Article

\title{
Active Pulmonary Tuberculosis in Elderly Patients: A 2016-2019 Retrospective Analysis from an Italian Referral Hospital
}

\author{
Francesco Di Gennaro ${ }^{1, *,+}{ }^{\circ}$, Pietro Vittozzi ${ }^{1,+}$, Gina Gualano ${ }^{1}$, Maria Musso ${ }^{1}$, Silvia Mosti ${ }^{1}$, \\ Paola Mencarini ${ }^{1}$, Carlo Pareo ${ }^{1}$, Antonino Di Caro ${ }^{2}{ }^{\mathbb{D}}$, Vincenzo Schininà ${ }^{3}$, Enrico Girardi ${ }^{4}$ \\ and Fabrizio Palmieri ${ }^{1}$ \\ 1 Respiratory Infectious Diseases Unit, National Institute for Infectious Diseases “L. Spallanzani” IRCCS, \\ 00149 Rome, Italy; pietro.vittozzi@inmi.it (P.V.); gina.gualano@inmi.it (G.G.); maria.musso@inmi.it (M.M.); \\ silvia.mosti@inmi.it (S.M.); paola.mencarini@inmi.it (P.M.); carlo.pareo@inmi.it (C.P.); \\ fabrizio.palmieri@inmi.it (F.P.) \\ 2 Microbiology and Bio-Repository Unit, National Institute for Infectious Diseases "L. Spallanzani" IRCCS, \\ 00149 Rome, Italy; antonino.dicaro@inmi.it \\ 3 Diagnostic Imaging Unit, National Institute for Infectious Diseases "L. Spallanzani” IRCCS, \\ 00149 Rome, Italy; vincenzo.schinina@inmi.it \\ 4 Clinical Epidemiology Unit, National Institute for Infectious Diseases “L. Spallanzani" IRCCS, \\ 00149 Rome, Italy; enrico.girardi@inmi.it \\ * Correspondence: francesco.digennaro@inmi.it; Tel.: +39-3924-804-707 \\ $\dagger$ These Authors contributed equally.
}

Received: 27 June 2020; Accepted: 5 August 2020; Published: 7 August 2020

\begin{abstract}
Tuberculosis (TB) in the elderly ( $>65$ years old) has increasingly become a global health problem. It has long been recognized that older people are vulnerable to developing tuberculosis. We retrospectively evaluated data from patients older than 65 years diagnosed with pulmonary TB admitted to the National Institute for Infectious Diseases L. Spallanzani, Rome, Italy, from 1 January 2016 to 31 December 2019. One hundred and six consecutive patients were diagnosed with pulmonary $\mathrm{TB}$ and $68 \%$ reported at least one comorbidity and $44 \%$ at least one of the TB risk-factors. Out of the 26 elderly patients who reported an adverse event, having risk factors for TB (O.R. (Odds Ratios) $=1.45 ; 95 \%$ CI 1.12-3.65) and the presence of cavities on Chest X-rays $(\mathrm{O} . \mathrm{R} .=1.42 ; 95 \% \mathrm{CI} 1.08-2.73)$ resulted in being more likely to be associated with adverse events in elderly patients. Having weight loss (O.R. $=1.31 ; 95 \%$ CI 1.08-1.55) and dyspnea (O.R. = 1.23; 95\% CI 1.13-1.41) resulted in being significant predictors of unsuccessful treatment outcome in elderly patients. Older people with TB represent a vulnerable group, with high mortality rate, with a challenging diagnosis. Hospitalizations in tertiary referral hospital with clinical expertise in TB management can be useful to improve the outcome of these fragile patients.
\end{abstract}

Keywords: tuberculosis (TB); elderly; outcome; adverse events; risk factors

\section{Introduction}

Despite extensive tuberculosis-control efforts of the World Health Organization (WHO) and local health departments, the tuberculosis (TB) epidemic continues to ravage the world, affecting susceptible individuals including the elderly ( $>65$ years old), and representing a global health problem [1,2]. The geriatric population in a high income setting such as in Italy represents a large reservoir of TB infection across all sexual and gender subgroups. Age-related comorbidities (e.g., malnutrition, cancer, chronic renal failure, and diabetes mellitus), together with physiological biological changes may weaken 
protective barriers, impair microbial clearance mechanisms, and contribute to reducing cellular immune responses to $M$. tuberculosis, thus increasing the risk of TB among this age group [3]. Moreover elderly people are both at especially high risk for reactivation of latent $\mathrm{TB}$, but also susceptible to new $\mathrm{TB}$ infection. Tuberculosis diagnosis in the elderly can be challenging; in fact, elderly patients may have an absent or attenuated febrile response with a high frequency of nonspecific clinical manifestations or co-morbidities and less frequently "classical" radiological presentations that can result in delay in diagnosis [4]. Older patients $\geq 65$ years of age have a higher mortality rate compared to patients under the age of 65 [5]. In fact, global data from low incidence countries show that around $80 \%$ of the deaths occur in patients over sixty-five years of age [6]. The mortality rates of older patients have been reported as up to 51\% [7]. Although these mortality rates have been decreasing recently, the rate remains high [8,9]. Moreover, age represents a risk factor connected to the development of adverse drug reactions due to polypharmacy, pill burden, existing co-morbidities, and a lower efficiency of renal and hepatic drugs clearance. $[6,10]$

To our knowledge, there are no data in Italy investigating and reporting findings from this vulnerable group. Therefore, we conducted a retrospective study in elderly pulmonary TB patients in order to describe clinical presentation and factors associated with adverse events and outcome.

\section{Results}

Between 1 January 2016 and 31 December 2019, 106 consecutive patients age $>65$ (median age years 76, IQR 5; male n. 73, 69\%) were diagnosed with pulmonary TB at INMI L. Spallanzani Institute in Rome and included in the study.

Table 1 shows the characteristics of participants overall and stratified by age-classes $\leq 75$ or $>75$ years old. Of the whole sample, $68 \%(n=73)$ reported at least one comorbidity, and $44 \%(n=47)$ at least one of the TB risk-factors between diabetes, chronic renal failure, malignances, and being under immunosuppressive therapy. Forty patients out of 106 (37\%) were sputum smear positive. Notably, $52 \%(n=55)$ of the participants referred non-specific initial symptoms, and $17 \%(n=18)$ also had an extrapulmonary localization of TB.

Adverse events related to TB drug regimen were reported in $24 \%(n=26)$. Ninety-one per cent ( $n=96)$ of patients successfully completed the treatment, while $8 \%(n=9)$ died due to a TB-related cause and one failed. No differences in distribution of the variables collected between $\leq 75$ or $>75$ year-old groups emerged ( $p$-value $>0.05$ ).

Out of the 26 elderly patients who reported an adverse event, $69 \%(n=18)$ showed liver disease. In 16 patients $(61 \%)$, the event required minimal non-invasive intervention classified as of moderate severity and $81 \%(n=21)$ of cases required a suspension of suspect drug and change of treatment. The new therapeutic scheme after suspension included fluoroquinolones in 15 out of 21 patients $(71 \%)$. Further characteristics of adverse events reported in the sample are shown in Table 2.

Adverse events related to TB drug regimen were reported in $24 \%(n=26)$. Ninety-one per cent $(n=96)$ of patients successfully completed the treatment, while $8 \%(n=9)$ died due to a TB-related cause and one failed. No differences in distribution of the variables collected between $\leq 75$ or $>75$ year-old groups emerged ( $p$-value $>0.05)$.

Out of the 26 elderly patients who reported an adverse event, $69 \%(n=18)$ showed liver disease. In 16 patients $(61 \%)$, the event required minimal non-invasive intervention classified as of moderate severity and $81 \%(n=21)$ of cases required a suspension of suspect drug and change of treatment. The new therapeutic scheme after suspension included fluoroquinolones in 15 out of 21 patients $(71 \%)$. Further characteristics of adverse events reported in the sample are shown in Table 2.

The multivariate model on adverse events considered the effects of age, gender, risk factors for TB, comorbidities, fever, cough, dyspnea, cavities on CXR, drug without $Z$ regimen including fluorquinolone, extrapulmonary TB, acid-fast bacilli smear positive, and TB culture. Significant predictors of adverse events are reported in Table 3. Having risk factors for TB (O.R. $=1.45 ; 95 \%$ CI 1.12-3.65) and presence of 
cavities on CXR (O.R. $=1.42 ; 95 \%$ CI 1.08-2.73) resulted in being more likely to be associated with adverse events in elderly patients.

The multivariate model on unsuccessful outcome considered the effects of age, gender, risk factors for $\mathrm{TB}$, comorbidities, fever, cough, dyspnea, weight loss, cavities on CXR, drug without $\mathrm{Z}$ regimen including fluorquinolone, extrapulmonary $\mathrm{TB}$, acid-fast bacilli smear positive, and culture positive. Having weight loss (O.R. $=1.31 ; 95 \%$ CI 1.08-1.55) and dyspnea (O.R. $=1.23 ; 95 \%$ CI 1.13-1.41) resulted in being significant predictors of unsuccessful treatment outcome (death or failure) in elderly patients, as reported in Table 4

Table 1. Characteristics of participants stratified by age-classes $\leq 75$ or $>75$ years old.

\begin{tabular}{|c|c|c|c|c|}
\hline \multirow[t]{2}{*}{ Characteristics } & $\begin{array}{l}\text { No. of Total } \\
\text { Participants }\end{array}$ & $\begin{array}{l}\text { No. of Participants } \\
\text { Aged } \leq 75\end{array}$ & $\begin{array}{l}\text { No. Of Participants } \\
\text { Aged }>75\end{array}$ & \multirow[t]{2}{*}{$p$-Value } \\
\hline & $106(100 \%)$ & $50(100 \%)$ & $56(100 \%)$ & \\
\hline \multicolumn{5}{|c|}{ Sex, N (\%) } \\
\hline Female & $33(31 \%)$ & $14(42 \%)$ & $19(58 \%)$ & \multirow{2}{*}{0.5} \\
\hline Male & $73(69 \%)$ & $36(49 \%)$ & $37(51 \%)$ & \\
\hline Age, median, (IQR) & $76(5)$ & $70.5(5)$ & $82(5)$ & 0.4 \\
\hline Comorbidities, n (\%) & $72(68 \%)$ & $32(64 \%)$ & $40(71 \%)$ & 0.4 \\
\hline $\begin{array}{c}\text { Risk Factors for TB, n (\%) } \\
\text { Type of diagnosis }(\%)\end{array}$ & $47(44 \%)$ & $19(40 \%)$ & $28(60 \%)$ & 0.2 \\
\hline Culture positive, $\mathrm{n}(\%)$ & $67(63 \%)$ & $28(56 \%)$ & $39(70 \%)$ & 0.3 \\
\hline NAT positive, $\mathrm{n}(\%)$ & $24(23 \%)$ & $10(20 \%)$ & $14(25 \%)$ & 0.4 \\
\hline Histological, $\mathrm{n}(\%)$ & $3(3 \%)$ & $2(4 \%)$ & $1(2 \%)$ & 0.5 \\
\hline Clinical, n (\%) & $12(11 \%)$ & $7(14 \%)$ & $5(9 \%)$ & 0.1 \\
\hline Monoresistance, $\mathrm{n}(\%)$ & $3(3 \%)$ & $3(6 \%)$ & $0(0 \%)$ & $\mathrm{Na}$ \\
\hline \multicolumn{5}{|c|}{ Initial TB symptoms, n (\%) } \\
\hline fever & $29(27 \%)$ & $16(32 \%)$ & $13(23 \%)$ & \\
\hline cough & $53(50 \%)$ & $26(52 \%)$ & $27(48 \%)$ & \\
\hline weight loss & $12(11 \%)$ & $8(16 \%)$ & $4(7 \%)$ & \\
\hline night sweats & $2(2 \%)$ & $1(2 \%)$ & $1(2 \%)$ & 0.2 \\
\hline hemoptysis & $9(8 \%)$ & $5(1 \%)$ & $4(7 \%)$ & \\
\hline dyspnea & $24(23 \%)$ & $10(20 \%)$ & $14(25 \%)$ & \\
\hline Non- specific symptoms * & $55(52 \%)$ & $22(44 \%)$ & $33(59 \%)$ & \\
\hline Cavities on CXR, n (\%) & $32(29 \%)$ & $15(30 \%)$ & $17(28 \%)$ & 0.8 \\
\hline $\begin{array}{l}\text { Concurrent } \\
\text { Extrapulmonary TB } \mathrm{n}(\%)\end{array}$ & $18(17 \%)$ & $8(16 \%)$ & $10(18 \%)$ & 0.8 \\
\hline \multicolumn{5}{|c|}{ Initial Therapeutic Scheme, n (\%) } \\
\hline $\mathrm{R}+\mathrm{H}+\mathrm{E}+\mathrm{Z}$ & $76(72 \%)$ & $40(80 \%)$ & $36(64 \%)$ & \multirow{3}{*}{0.4} \\
\hline $\begin{array}{l}\text { Drug regimen without } Z \\
\text { including Amikacin }\end{array}$ & $4(3 \%)$ & $0(\%)$ & $4(7 \%)$ & \\
\hline $\begin{array}{l}\text { Drug without } Z \text { regimen } \\
\text { including fluoroquinolone }\end{array}$ & $26(24 \%)$ & $11(22 \%)$ & $15(26 \%)$ & \\
\hline $\begin{array}{c}\text { Adverse events, n (\%) } \\
\text { Outcomes, } \mathrm{n}(\%)\end{array}$ & $26(24 \%)$ & $15(30 \%)$ & $11(20 \%)$ & 0.5 \\
\hline successful treatment & $96(91 \%)$ & $47(94 \%)$ & $49(87 \%)$ & \\
\hline died & $9(8 \%)$ & $2(4 \%)$ & $7(13 \%)$ & 0.2 \\
\hline failure & $1(1 \%)$ & $1(2 \%)$ & $0(0 \%)$ & \\
\hline
\end{tabular}

${ }^{*}$ non-specific symptoms: headache, gastrointestinal. 
Table 2. Characteristics of adverse events in the 26 patients who reported them.

\begin{tabular}{|c|c|}
\hline \multirow{2}{*}{ Characteristics } & Total \\
\hline & $26(100)$ \\
\hline & $\mathbf{N}(\%)$ \\
\hline \multicolumn{2}{|l|}{ Type of Adverse events, $\mathrm{n}(\%)$} \\
\hline Hepatitis & $18(69 \%)$ \\
\hline Ocular damage/ decrease in visual acuity & $2(7 \%)$ \\
\hline itching / skin rash & $3(11 \%)$ \\
\hline arrhythmia & $1(4 \%)$ \\
\hline gastrointestinal & $3(11 \%)$ \\
\hline acute renal failure & $1(4 \%)$ \\
\hline More than 1 Adverse events, $n(\%)$ & $3(11 \%)$ \\
\hline \multicolumn{2}{|l|}{ Severity of Adverse events, $\mathrm{n}(\%)$} \\
\hline Mild & $4(15 \%)$ \\
\hline Moderate & $16(61 \%)$ \\
\hline Severe & $6(23 \%)$ \\
\hline \multicolumn{2}{|l|}{ Adverse events management, $n(\%)$} \\
\hline temporary suspension of suspect drug and change of drug & $18(69 \%)$ \\
\hline temporary suspension of all treatment & $1(4 \%)$ \\
\hline definitive suspension of suspect drug & $2(8 \%)$ \\
\hline support therapy and no change of treatment & $5(19 \%)$ \\
\hline \multicolumn{2}{|l|}{ Which drugs, n (\%) } \\
\hline $\mathrm{E}$ & $1(4 \%)$ \\
\hline $\mathrm{H}$ & $3(11 \%)$ \\
\hline $\mathrm{Z}$ & $10(38 \%)$ \\
\hline Mfx & $1(4 \%)$ \\
\hline $\begin{array}{c}>1 \text { drugs } \\
\text { (of which } 8 / 11 \mathrm{Z}+\mathrm{H} \text { ) }\end{array}$ & $11(42 \%)$ \\
\hline \multicolumn{2}{|l|}{ 2nd therapeutic scheme, n (\%) } \\
\hline With FQ & $15(71 \%)$ \\
\hline Without FQ & $6(29 \%)$ \\
\hline
\end{tabular}

Table 3. Predictors of adverse events for active pulmonary tuberculosis in elderly patients.

\begin{tabular}{ccc}
\hline Characteristics & Univariate Analysis O.R. & Multivariate Analysis Adj-O.R. \\
\hline Age $<75$ & $1.02(0.98-1.04)$ & $0.94(0.77-2.11)$ \\
Female & $0.28(0.16-0.40)$ & $0.58(0.27-1.78)$ \\
Risk Factors for TB & $1.30(1.18-1.54)$ & $1.45(1.12-3.65)^{*}$ \\
Comorbidities & $0.54(0.10-1.10)$ & $0.49(0.28-3.08)$ \\
Fever & $1.21(0.28-1.23)$ & $1.33(1.18-1.54)$ \\
Cough & $0.64(0.38-0.78)$ & $0.74(0.50-1.03)$ \\
Dyspnea & $1.26(0.85-1.72)$ & $1.33(1.17-3.90)$ \\
Cavities on CXR & $1.20(0.09-1.48)$ & $1.42(1.08-2.73) *$ \\
Regimen without Z regimen & $0.25(0.10-0.40)$ & $0.78(0.45-1.74)$ \\
including fluorquinolone & $0.79(0.68-1.21)$ & $1.01(0.83-3.21)$ \\
R + H + E + Z (Standard regimen) & $0.57(0.59-0.83)$ & $0.51(0.43-2.70)$ \\
Extrapulmonary TB & $1.06(0.25-1.09)$ & - \\
Acid-fast bacilli smear positive & $1.21(0.89-1.73)$ & - \\
Tb culture positive &
\end{tabular}


Table 4. Predictors of unsuccessful treatment outcome in elderly TB patients.

\begin{tabular}{ccc}
\hline Characteristics & Univariate Analysis O.R & Multivariate Analysis Adj-O.R \\
\hline Age $<75$ & $0.44(0.29-2.08)$ & $0.55(0.26-1.06)$ \\
Female & $0.68(0.41-0.74)$ & $0.63(0.43-1.61)$ \\
Risk Factors for TB & $1.18(0.81-6.47)$ & $1.36(0.80-2.90)$ \\
Comorbidities & $0.90(0.69-1.21)$ & $1.19(0.10-1.40)$ \\
Adverse events & $1.03(0.88-1.30)$ & $1.18(0.71-1.25)$ \\
Fever & $1.01(0.94-1.95)$ & $1.40(0.86-1.61)$ \\
Cough & $0.87(0.36-1.71)$ & $0.73(0.50-1.91)$ \\
Weight loss & $1.28(1.10-1.44)$ & $1.31(1.08-1.55)^{*}$ \\
Dyspnea & $1.18(1.03-1.74)$ & $1.23(1.13-1.41)^{*}$ \\
Cavities on CXR & $1.20(1.03-1.27)$ & $1.48(0.54-1.75)$ \\
Drug regimen without Z & $1.30(0.99-2.77)$ & - \\
including Fluoroquinolone & $0.88(0.76-1.09)$ & $1.08(0.78-1.71)$ \\
R + H + E + Z & $0.65(0.28-0.85)$ & $1.54(0.34-3.92)$ \\
Extrapulmonary TB & $0.51(0.33-2.61)$ & - \\
Acid-fast bacilli smear positive & $1.45(0.38-1.77)$ & $0.65(0.28-2.68)$ \\
TB culture positive & statist &
\end{tabular}

* statistically significant.

\section{Discussion}

Our cohort describes a large group of elderly patients from a tertiary referral hospital for TB in Italy. According to the WHO definitions, in our cohort, 91 out of 106 patients (86\%) were bacteriologically confirmed with pulmonary TB.

As highlighted by the $\mathrm{WHO}, \mathrm{TB}$ in elderly patients represents one of the pilot keys for high income countries to control TB burden [11-13]. TB and age are strongly related since underlying illness and the physiological reduction of the immune system of the elderly make this group of people more susceptible to a reactive latent form or to a new infection $[14,15]$.

Previous data showed that co-morbidities such as diabetes, COPD, and cardiac disease, which are prevalent in aging populations, increase the risk of developing active TB disease [16,17]. In this cohort, more than half of the patients had one or more comorbidities, and presented risk factors for developing TB. Despite age and comorbidities, 96 (91\%) patients were defined as successfully treated. Unsuccessful outcome was reported in 10 out of 106 patients (9\%), where nine patients died.

Unlike other studies, there was no difference in unsuccessful treatment between age $<75$ years and $>75$ years in TB patients and the mortality rate was only 8.5\% (9/106) in older patients [18]. Our data showed lower mortality rates than reported in other studies worldwide (up to 51\%) $[19,20]$ and in European low incidence countries (from 11.2\% in UK to 29.3 in Spain) $[8,21]$.

We believe that the low mortality and high treatment success rates in this retrospective study could reflect the accurate diagnosis, coupled with the opportunity to test for drug susceptibility, timely monitoring, and treatment of adverse events, and close follow up after discharge. Moreover, two elements can have contributed: the Italian health system, which provides universal coverage to citizens and residents free of charge, and the setting of the study, a tertiary referral hospital with clinical expertise in the diagnosis and treatment of TB. Furthermore, our experience confirmed, according to the WHO statements, the role of active TB drug-safety monitoring, aDSM, especially in the elderly, to minimize the unsuccessful treatment [22,23].

However, the data findings underline the vulnerability of these patients, the difficulty in achieving therapeutic success compared to other categories, and the need for clinical attention.

Two recent meta-analyses investigated the factors associated with a worse treatment outcome. The authors found that age, sex, alcohol consumption, smoking, sputum smear non-conversion at two months of treatment, and HIV affect the results of TB treatment. From their research, no clinical data influenced TB outcome [24,25]. 
In our cohort, $52 \%(n=55)$ of the participants referred non-specific initial symptoms, and $17 \%$ $(n=18)$ also had an extrapulmonary localization of TB. We found that weight loss and dyspnea at diagnosis were negative predictive factors for negative outcome.

Diagnostic difficulties in the elderly are common in many diseases, not solely TB, and the development of new diagnostic tools such as ultrasound and point of care diagnostic can help overcome this important issue [26,27].

Many papers have been published with regard to clinical and radiologic presentation of TB in varying ages. Patients may present a lack of respiratory symptoms and may be unable to expectorate sputum due to weakness. In a comparison between clinical features in the young and old (adults), the classical symptoms of productive cough, night sweats, fever, weight loss, and hemoptysis were much less common in the older age group [28,29].

In our cohort, fever, cough, dyspnea, and weight loss were the most common symptoms. We found that dyspnea and weight loss at the time of diagnosis were predictive factors for negative outcome. Dyspnea and weight loss could have been associated with a more advanced stage of the disease, which has been more affected by diagnostic delay, and with poorer clinical conditions. Many studies demonstrated how advanced stages of illness were associated with unfavorable outcome, underlining the pivot role of early recognition of symptoms and rapid diagnosis and therapy for TB outcome [30].

Radiological findings represent another challenging diagnosis element [31,32]. Imaging could provide suspects for diagnosis and timely treatment for active TB. Consistently with other data in literature, only 32 patients (29\%) showed typical radiographs of upper lobe cavitary TB lesions, while another study showed a smaller proportion $(16 \%)$ of patients with typical radiological manifestations [33].

Adverse events (AEs) represent an important issue in a long therapy like TB. In our cohort, $26(24 \%)$ patients developed AEs (six were severe AE); the most common was liver disfunction (69\%).

Elderly people are more likely to develop adverse drug interaction from polypharmacy, pill burden, existing co-morbidities, and age-related physiological changes. Many reasons can explain the high rate of adverse events: active research by clinicians of adverse events during hospitalization or outpatient controls, hepatotoxicity from anti-tuberculous drugs rises significantly with increasing age, visual impairment, poor memory, and reduced mobility may cause poor adherence to the drug regimen $[34,35]$.

From our data, predictors of adverse events for TB in elderly patients were: having risk factors for TB (diabetes, malnutrition, alcohol abuse, immunosuppressive therapy) and cavities on CXR. Additionally, other studies have shown how risk factors to onset TB were independent predictors of adverse drug events. Thus, closely monitored follow-ups are highly recommended in these patients [36,37].

Consistent with the other data in the literature, our study identified Pyrazinamide and Isoniazid as drugs responsible for AEs [38-40].

Contrary to other studies, no mortality can be attributed to drugs [41]. Discontinuation of suspected drugs required a change in treatment regimen in 21 patients who developed AEs.

In 26 patients, first line regimen was replaced by fluoroquinolones (FQs) and in 15 patients, FQs were added after suspension due to AEs. No significant AEs was found in patients receiving FQs. International warnings have recommended that it is used cautiously in elderly patients and those with renal impairment due to possible QTc prolongation, increased risk for drug accumulation, and in patients with diabetes or those who are taking hypoglycemic agents due to the risk for severe hypoglycemia [42,43]. In our cohort, 41 patients (39\%) were on fluoroquinolones. We found only one cardiac adverse event (arrhythmia). Despite other evidence, in our experience, FQs were well tolerated drugs [44].

We recognize some limitations in our study: the enrolment of patients diagnosed in one institution may limit the extent to which our findings can be generalized. The retrospective nature of the 
study precluded the consideration of other factors potentially influencing outcome such as lack of socioeconomic characteristics and evaluation of diagnostic delay [25].

\section{Materials and Methods}

\subsection{Study Design and Patients}

This is a retrospective study in elderly Italians diagnosed with pulmonary TB admitted to the National Institute for Infectious Diseases L. Spallanzani, Rome, Italy, from 1 January 2016 to 31 December 2019. The study protocol was approved by L. Spallanzani Institute Ethics Committee (Decision No. 12/2015)

We retrospectively evaluated data from patients older than 65 years old with pulmonary active TB. According to the WHO guidelines, enrolled patients were classified as "active pulmonary TB" if the diagnosis was based: (i) on a positive culture for $M$. tuberculosis from a respiratory sample (sputum or bronchoalveolar lavage) or other biological specimens; (ii) on positive M. tuberculosis nucleic amplification test NAT (TRCReady ${ }^{\circledR}$ MTB, Tosoh Bioscience, Japan or Xpert ${ }^{\circledR}$ MTB/RIF, Cepheid, USA) from biological specimens (without culture confirmation); or (iii) on histopathological findings consistent with TB and presence of acid fast bacilli (AFB) in a tissue sample. Moreover, patients were classified as "clinical TB" if the diagnosis was based on clinical and radiologic criteria (having excluded other diseases) including appropriate response to standard anti-TB therapy.

Patients were treated according to the institutional protocol drawn up following the WHO TB guidelines [11]. Initial treatment was provided on an in-hospital basis, until AFB sputum conversion was achieved on three consecutive negative samples collected during one week. Patients received direct observed therapy during hospitalization. After discharge, patients were followed monthly on ambulatory care by trained TB specialists for the full course of treatment. Laboratory tests were repeated almost monthly, or as needed according to clinical condition, until the treatment was completed. Medical counseling was given regarding the possibility of adverse drug reaction occurrence and the importance of reporting any adverse event to their physician as soon as it presented. Patients were also provided with an information sheet about the most common side effects of anti-TB drugs.

\subsection{Data Collection}

The primary data source was the patient chart, from where a set of predefined variables were retrospectively collected by a study physician and included patient demographics; admission and discharge/death date; and clinical variables: symptoms, comorbidities, risk factors for TB, TB diagnosis, M. tuberculosis drug resistance, chest x-ray (CXR) findings, TB localization, treatment regimen, adverse events (type, severity, management), and outcomes. As comorbidities, previous TB, COPD/bronchiectasis, cardiopathy, hypothyroidism, dementia, hematological disease, and chronic liver disease were included. Risk factors for TB included diabetes, chronic renal failure, malignances, and being under immunosuppressive therapy.

Severity of adverse events were classified as mild (asymptomatic laboratory findings only; minor signs/symptoms; no medical intervention required), moderate (requiring minimal non-invasive intervention), and severe (significant symptoms requiring hospitalization) [11].

\subsection{Statistical Analysis}

No formal sample size was calculated a priori, and the study included all patients older than 65 years admitted during the study period. Continuous data were expressed as median and interquartile range (IQR), and categorical data as numbers and percentages. X2 test (with Fisher's correction as required) was used to compare categorical variables. We stratified our cohort into two groups: $<75$ years of age and older than 75 years in order to verify any differences between these two groups.

Two logistic regression models were implemented considering the dependent variables as: (I) the adverse events; and (II) unsuccessfully treated (died and failed patients) while each of the available 
factors were considered as independent variables (univariate analysis). In the multivariate analysis, all the factors with a $p$-value $<0.10$ at the univariate analyses were included. Multicollinearity among covariates was assessed through the variance inflation factor (VIF), taking a value of two for excluding a covariate. However, no variable was excluded according to the previous criterion.

Odds ratios (ORs) as adjusted odds ratios (Adj-ORs) with their 95\% confidence intervals (CIs) were used to measure the association between factors at the baseline (exposure) and treatment failure (outcome).

All analyses were 2-sided and a $p$-value less than 0.05 was considered statistically significant. Statistical analysis was performed using STATA V.13.

\section{Conclusions}

In conclusion, our data suggest that older people with TB represent a vulnerable group with high mortality rate, a challenging diagnosis due to a higher frequency of atypical features, symptoms common with other age-related diseases, and more frequent adverse drug reactions. Dyspnea and weight loss at $\mathrm{TB}$ diagnosis are risk factors for treatment failure and describe the phenotype of a patient who needs more medical attention and close monitoring. Having risk factors for TB also represents a predictor for adverse events. In addition, data suggest the pivotal role of aDSM to improve outcome in older patients. Hospitalization in tertiary referral hospital with clinical expertise in diagnosis and treatment of TB can be useful to improve outcome considering the elderly as fragile patients. New treatment regimens and novel diagnostics, aiming to reduce adverse events of treatment in TB, should also all be specifically evaluated in elderly populations to reduce pill burden, and increase outcome and safety. TB management, control program, and global research agenda need to consider these patients as a possible key to control TB, especially in low burden countries.

Author Contributions: Conceptualization, F.P., G.G., and E.G.; Methodology, F.D.G. and F.P.; Validation, F.D.G., P.V., G.G., M.M., S.M., P.M., C.P., A.D.C., V.S., E.G. and F.P.; Formal writing-original draft preparation, F.D.G., G.G. and F.P., Writing-review and editing, E.G., C.P., M.M., S.M., P.V. and V.S. All authors have read and agreed to the published version of the manuscript.

Funding: This study was supported by funding from the Italian Ministry of Health, grant Ricerca Corrente IRCCS Research program n.4 Tuberculosis. The funders had no role in the study design, data collection, and analysis, decision to publish, or preparation of the manuscript.

Acknowledgments: We sincerely thank E. Ercoli and all the nursing staff and socio-health operators of the Respiratory Infectious Diseases Unit, who provided expertise and care for the patients.

Conflicts of Interest: The authors declare no conflicts of interest.

\section{References}

1. Schaaf, H.S.; Collins, A.; Bekker, A.; Davies, P.D. Tuberculosis at extremes of age. Respirology 2010, 15, 747-763. [CrossRef] [PubMed]

2. Kwon, Y.-S.; Chi, S.Y.; Oh, I.-J.; Kim, K.-S.; Kim, Y.I.; Lim, S.C.; Kim, Y.-C. Clinical characteristics and treatment outcomes of tuberculosis in the elderly: A case control study. BMC Infect. Dis. 2013, 13, 121. [CrossRef] [PubMed]

3. Aw, D.; Silva, A.B.; Palmer, D.B. Immunosenescence: Emerging challenges for an ageing population. Immunology 2007, 120, 435-446. [CrossRef] [PubMed]

4. Rajagopalan, S. Tuberculosis and Aging: A Global Health Problem. Clin. Infect. Dis. 2001, 33, $1034-1039$. [CrossRef]

5. Cruz-Hervert, L.P.; García-García, L.; Ferreyra-Reyes, L.; Valle, M.B.-D.; Cano-Arellano, B.; Canizales-Quintero, S.; Ferreira-Guerrero, E.; Báez-Saldaña, R.; Téllez-Vázquez, N.; Nava-Mercado, A.; et al. Tuberculosis in ageing: High rates, complex diagnosis and poor clinical outcomes. Age Ageing 2012, 41, 488-495. [CrossRef]

6. WHO. Framework towards Tuberculosis Elimination in Low-Incidence Countries; WHO: Geneva, Switzerland, 2014.

7. Davies, P.D. TB in the elderly in industrialised countries. Int. J. Tuberc. Lung Dis. 2007, 11, 1157-1159.

8. Pratt, R.H.; Winston, C.A.; Kammerer, J.S.; Armstrong, L.R. Tuberculosis in older adults in the United States, 1993-2008. J. Am. Geriatr. Soc. 2011, 59, 851-857. [CrossRef] 
9. Min, J.; Kim, J.S.; Kim, H.W.; Shin, A.Y.; Koo, H.-K.; Lee, S.-S.; Kim, Y.-K.; Shin, K.-C.; Chang, J.H.; Chun, G.; et al. Clinical profiles of early and tuberculosis-related mortality in South Korea between 2015 and 2017: A cross-sectional study. BMC Infect. Dis. 2019, 19, 735. [CrossRef]

10. Di Gennaro, F.; Marotta, C.; Antunes, M.; Pizzol, D. Diabetes in active tuberculosis in low-income countries: To test or to take care? Lancet Glob. Health 2019, 7, e707. [CrossRef]

11. World Health Organization. Guidelines on Tuberculosis Infection Prevention and Control, 2019 Update; WHO/CDS/TB/2019.1 License: CC BY-NC-SA 3.0 IGO; World Health Organization: Geneva, Switzerland, 2019. Available online: https:/apps.who.int/iris/bitstream/handle/10665/311259/9789241550512-eng.pdf?ua=1 (accessed on 30 May 2020).

12. Lönnroth, K.; Migliori, G.B.; Abubakar, I.; D'Ambrosio, L.; De Vries, G.; Diel, R.; Douglas, P.; Falzon, D.; Gaudreau, M.-A.; Goletti, D.; et al. Towards tuberculosis elimination: An action framework for low-incidence countries. Eur. Respir. J. 2015, 45, 928-952. [CrossRef]

13. World Health Organization. Towards Tuberculosis Elimination: An Action Framework for Low-Incidence Countries; World Health Organization: Geneva, Switzerland, 2014.

14. Piergallini, T.J.; Turner, J. Tuberculosis in the elderly: Why inflammation matters. Exp. Gerontol. 2018, 105, 32-39. [CrossRef] [PubMed]

15. Byng-Maddick, R.; Noursadeghi, M. Does tuberculosis threaten our ageing populations? BMC Infect. Dis. 2016, 16, 119. [CrossRef] [PubMed]

16. Inghammar, M.; Ekbom, A.; Engström, G.; Ljungberg, B.; Romanus, V.; Löfdahl, C.-G.; Egesten, A. COPD and the Risk of Tuberculosis-A Population-Based Cohort Study. PLoS ONE 2010, 5, e10138. [CrossRef] [PubMed]

17. Pizzol, D.; Di Gennaro, F.; Chhaganlal, K.D.; Fabrizio, C.; Monno, L.; Putoto, G.; Saracino, A. Prevalence of diabetes mellitus in newly diagnosed pulmonary tuberculosis in Beira, Mozambique. Afr. Health Sci. 2017, 17, 773-779. [CrossRef]

18. Salvadó, M.; Garcia-Vidal, C.; Vázquez, P.; Nr, M.R.; Carballeira, M.R.; Martínez-Lacasa, J.; Cuchí, E.; Garau, J.; Martínez-Lacasa, J. Mortality of Tuberculosis in Very Old People. J. Am. Geriatr. Soc. 2010, 58, 18-22. [CrossRef]

19. Oshi, D.C.; Oshi, S.N.; Alobu, I.; Ukwaja, K.N. Profile and Treatment Outcomes of Tuberculosis in the Elderly in Southeastern Nigeria, 2011-2012. PLoS ONE 2014, 9, e111910. [CrossRef]

20. Pizzol, D.; Veronese, N.; Marotta, C.; Di Gennaro, F.; Moiane, J.; Chhaganlal, K.; Monno, L.; Putoto, G.; Mazzucco, W.; Saracino, A. Predictors of therapy failure in newly diagnosed pulmonary tuberculosis cases in Beira, Mozambique. BMC Res. Notes 2018, 11, 99. [CrossRef]

21. Abbara, A.; Collin, S.; Kon, O.M.; Buell, K.; Sullivan, A.; Barrett, J.; Corrah, T.; McGregor, A.; Hansel, T.; John, L.; et al. Time to diagnosis of tuberculosis is greater in older patients: A retrospective cohort review. ERJ Open Res. 2019, 5. [CrossRef]

22. World Health Organization Active TB Drug-Safety Monitoring and Management (aDSM); World Health Organization: Geneva, Switzerland, 2015.

23. Borisov, S.; Danila, E.; Maryandyshev, A.; Dalcolmo, M.; Miliauskas, S.; Kuksa, L.; Manga, S.; Skrahina, A.; Diktanas, S.; Codecasa, L.R.; et al. Surveillance of adverse events in the treatment of drug-resistant tuberculosis: First global report. Eur. Respir. J. 2019, 54, 1901522. [CrossRef]

24. Torres, N.M.C.; Rodríguez, J.J.Q.; Andrade, P.S.P.; Arriaga, M.; Netto, E.M. Factors predictive of the success of tuberculosis treatment: A systematic review with meta-analysis. PLoS ONE 2019, 14, e0226507. [CrossRef]

25. Di Gennaro, F.; Pizzol, D.; Cebola, B.; Stubbs, B.; Monno, L.; Saracino, A.; Luchini, C.; Solmi, M.; Segafredo, G.; Putoto, G.; et al. Social determinants of therapy failure and multi drug resistance among people with tuberculosis: A review. Tuberculosis 2017, 103, 44-51. [CrossRef] [PubMed]

26. García-Basteiro, A.; Dinardo, A.; Saavedra, B.; Silva, D.; Palmero, D.; Gegia, M.; Migliori, G.; Duarte, R.; Mambuque, E.; Centis, R.; et al. Point of care diagnostics for tuberculosis. Pulmonology 2018, 24, 73-85. [CrossRef] [PubMed]

27. Bobbio, F.; Di Gennaro, F.; Marotta, C.; Kok, J.; Akec, G.; Norbis, L.; Monno, L.; Saracino, A.; Mazzucco, W.; Lunardi, M. Focused ultrasound to diagnose HIV-associated tuberculosis (FASH) in the extremely resource-limited setting of South Sudan: A cross-sectional study. BMJ Open 2019, 9, e027179. [CrossRef] [PubMed]

28. Pérez-Guzmán, C.; Vargas, M.H.; Torres-Cruz, A.; Villarreal-Velarde, H. Does Aging Modify Pulmonary Tuberculosis? Chest 1999, 116, 961-967. [CrossRef] 
29. Li, J.; Chung, P.H.; Leung, C.L.K.; Nishikiori, N.; Chan, E.Y.Y.; Yeoh, E.K. The strategic framework of tuberculosis control and prevention in the elderly: A scoping review towards End TB targets. Infect. Dis. Poverty 2017, 6, 70, Published 1 June 2017. [CrossRef]

30. Guthmann, J.-P.; Léon, L.; Antoine, D.; Lévy-Bruhl, D. Tuberculosis treatment outcomes of notified cases: Trends and determinants of potential unfavourable outcome, France, 2008 to 2014 . Eurosurveillance 2020, 25, 1900191. [CrossRef]

31. Morris, C.D. Pulmonary tuberculosis in the elderly: A different disease? Thorax 1990, 45, 912-913. [CrossRef]

32. Di Gennaro, F.; Pisani, L.; Veronese, N.; Pizzol, D.; Lippolis, V.; Saracino, A.; Monno, L.; Huson, M.A.M.; Copetti, R.; Putoto, G.; et al. Potential Diagnostic Properties of Chest Ultrasound in Thoracic Tuberculosis-A Systematic Review. Int. J. Environ. Res. Public Health 2018, 15, 2235. [CrossRef]

33. Alavi, S.M.; Bakhtyarinia, P.; Hematnia, F.; Albagi, A. Clinical and Radiographic Manifestations and Treatment Outcome of Pulmonary Tuberculosis in the Elderly in Khuzestan, Southwest Iran. Tanaffos 2014, 13, 14-19.

34. LaVan, A.H.; Gallagher, P. Predicting risk of adverse drug reactions in older adults. Ther. Adv. Drug Saf. 2015, 7, 11-22. [CrossRef]

35. Di Gennaro, F.; Marotta, C.; Pizzol, D.; Chhaganlal, K.; Monno, L.; Putoto, G.; Saracino, A.; Casuccio, A.; Mazzucco, W. Prevalence and Predictors of Malaria in Human Immunodeficiency Virus Infected Patients in Beira, Mozambique. Int. J. Environ. Res. Public Health 2018, 15, 2032. [CrossRef] [PubMed]

36. Merid, M.W.; Gezie, L.D.; Kassa, G.M.; Muluneh, A.G.; Akalu, T.Y.; Yenit, M.K. Incidence and predictors of major adverse drug events among drug-resistant tuberculosis patients on second-line anti-tuberculosis treatment in Amhara regional state public hospitals; Ethiopia: A retrospective cohort study. BMC Infect. Dis. 2019, 19, 286. [CrossRef] [PubMed]

37. Gualano, G.; Mencarini, P.; Musso, M.; Mosti, S.; Murachelli, S.; Cannas, A.; Di Caro, A.; Goletti, D.; Girardi, E.; Palmieri, F. Putting in Harm to cure: Drug related adverse events do not affect outcome of patients receiving treatment for Multidrug-Resistant Tuberculosis. Respir. Infect. 2019, 54. [CrossRef]

38. Lin, H.-S.; Cheng, C.-W.; Lin, M.-S.; Chou, Y.-L.; Chang, P.-J.; Lin, J.-C.; Ye, J.-J. The clinical outcomes of oldest old patients with tuberculosis treated by regimens containing rifampicin, isoniazid, and pyrazinamide. Clin. Interv. Aging 2016, 11, 299-306. [CrossRef]

39. Tweed, C.D.; Wills, G.H.; Crook, A.M.; Dawson, R.; Diacon, A.H.; Louw, C.E.; McHugh, T.D.; Mendel, C.M.; Meredith, S.K.; Mohapi, L.; et al. Liver toxicity associated with tuberculosis chemotherapy in the REMoxTB study. BMC Med. 2018, 16, 46. [CrossRef]

40. Van Hest, R.; Baars, H.; Kik, S.; Van Gerven, P.; Trompenaars, M.-C.; Kalisvaart, N.; Keizer, S.; Borgdorff, M.; Mensen, M.; Cobelens, F. Hepatotoxicity of Rifampin-Pyrazinamide and Isoniazid Preventive Therapy and Tuberculosis Treatment. Clin. Infect. Dis. 2004, 39, 488-496. [CrossRef]

41. Hagiwara, E.; Suido, Y.; Asaoka, M.; Katano, T.; Okuda, R.; Sekine, A.; Kitamura, H.; Baba, T.; Komatsu, S.; Ogura, T. Safety of pyrazinamide-including regimen in late elderly patients with pulmonary tuberculosis: A prospective randomized open-label study. J. Infect. Chemother. 2019, 25, 1026-1030. [CrossRef]

42. Stahlmann, R.; Lode, H. Safety Considerations of Fluoroquinolones in the Elderly. Drugs Aging 2010, 27, 193-209. [CrossRef]

43. Lee, C.-C.; Lee, M.-T.G.; Hsieh, R.; Porta, L.; Lee, W.-C.; Lee, S.-H.; Chang, S.-S. Oral Fluoroquinolone and the Risk of Aortic Dissection. J. Am. Coll. Cardiol. 2018, 72, 1369-1378. [CrossRef]

44. Liu, H.H. Safety profile of the fluoroquinolones: Focus on levofloxacin. Drug Saf. 2010, 33, 353-369. [CrossRef]

(C) 2020 by the authors. Licensee MDPI, Basel, Switzerland. This article is an open access article distributed under the terms and conditions of the Creative Commons Attribution (CC BY) license (http://creativecommons.org/licenses/by/4.0/). 